\title{
Rogue wave modes for a derivative nonlinear Schrödinger model
}

\author{
Hiu Ning Chan, ${ }^{1}$ Kwok Wing Chow,,${ }^{1, *}$ David Jacob Kedziora, ${ }^{2}$ Roger Hamilton James Grimshaw, ${ }^{3}$ and Edwin Ding ${ }^{4}$ \\ ${ }^{1}$ Department of Mechanical Engineering, University of Hong Kong, Pokfulam, Hong Kong \\ ${ }^{2}$ Research School of Physics and Engineering, Australian National University, Canberra, Australian Capital Territory 0200, Australia \\ ${ }^{3}$ Department of Mathematical Sciences, Loughborough University, Loughborough LE11 3TU, United Kingdom \\ ${ }^{4}$ Department of Mathematics and Physics, Azusa Pacific University, Azusa, California 91702, USA \\ (Received 8 October 2013; revised manuscript received 14 January 2014; published 17 March 2014)
}

\begin{abstract}
Rogue waves in fluid dynamics and optical waveguides are unexpectedly large displacements from a background state, and occur in the nonlinear Schrödinger equation with positive linear dispersion in the regime of positive cubic nonlinearity. Rogue waves of a derivative nonlinear Schrödinger equation are calculated in this work as a long-wave limit of a breather (a pulsating mode), and can occur in the regime of negative cubic nonlinearity if a sufficiently strong self-steepening nonlinearity is also present. This critical magnitude is shown to be precisely the threshold for the onset of modulation instabilities of the background plane wave, providing a strong piece of evidence regarding the connection between a rogue wave and modulation instability. The maximum amplitude of the rogue wave is three times that of the background plane wave, a result identical to that of the Peregrine breather in the classical nonlinear Schrödinger equation model. This amplification ratio and the resulting spectral broadening arising from modulation instability correlate with recent experimental results of water waves. Numerical simulations in the regime of marginal stability are described.
\end{abstract}

DOI: 10.1103/PhysRevE.89.032914

PACS number(s): 05.45.Yv, 02.30.Jr, 47.35.Fg

\section{INTRODUCTION}

In fluid dynamics, nonlinear optics, and several other physical contexts, rogue waves (also known as freak waves and by a variety of other names) are unexpectedly large, spatially and temporally localized displacements from a background state. In the context of water waves, rogue waves are difficult to observe in the ocean and are challenging entities to study experimentally $[1,2]$. However, they are of practical as well as scientific interest, as they can be disastrous for marine shipping [3] and offshore structures.

The nonlinear Schrödinger (NLS) equation, which describes the slow evolution of a weakly nonlinear wave packet in deep water [4,5], has been employed widely as a simple analytical model [6,7]. In particular, the Peregrine breather solution of the NLS equation has been invoked as a model of a rogue wave, as it is the simplest elementary solution that is both spatially and temporally localized. However, because rogue waves can be strongly nonlinear, it is desirable to consider the consequences of adding higher-order nonlinear self-steepening terms, along with an appropriate higher-order linear dispersive term. This leads to a modified NLS model, also known as the Dysthe equation [1,8]. In particular, it is desirable to know if the breather solutions analogous to the Peregrine breather survive under the addition of such terms and if so, how their properties are changed.

While experiments in water waves might not be easy to conduct due to the difficulty in controlling the dispersion, optical settings have proven to be much more promising. In photonics, the NLS equation governs the dynamics of a temporal pulse in the setting of a fiber and is used frequently in the physics of spatial waveguides as well. Indeed, optical rogue waves have been observed in microstructured fibers, near

\footnotetext{
*Corresponding author: FAX: (852)2858-5415; kwchow@hku.hk
}

the threshold of soliton-fission supercontinuum generation [9]. Additional physical effects to extend the regime of validity of NLS models for these optical pulses, e.g., third-order dispersion and self-steepening nonlinearity, have also been incorporated [10].

When these higher-order physical effects, such as those arising in the Dysthe equation for water waves, are included in the dynamics of a nonlinear wave packet, the resulting modified NLS equation can contain a variety of extra terms. Here we focus on only one such term, associated with selfsteepening effects. In the simplest situation where that entity constitutes the only nonlinear term, a derivative NLS equation will result. In a general theoretical setting, several versions of derivative NLS equations have been studied in the literature. Two widely used models are the Chen-Lee-Liu (CLL) equation [11]

$$
i u_{t}+u_{x x}+i u u^{*} u_{x}=0
$$

(where the asterisk denotes the complex conjugate) and the Kaup-Newell (KN) equation [12]

$$
i u_{t}+u_{x x}+i\left(u^{2} u^{*}\right)_{x}=0 .
$$

The KN equation arises in the context of temporal pulses along optical fibers, associated with the physical phenomenon of self-steepening [13]. For water waves, the CLL equation with the inclusion of the usual cubic nonlinear term can be regarded as a simple approximation of the Dysthe equation for higher-order water wave theory, when the coupling to the induced mean flow is neglected [1]. Further discussion and correlation with recent experimental results will be given in Sec. V. It has been established that the KN and CLL equations are connected by a gauge transformation [14]. Strictly speaking, self-steepening nonlinearity in optics refers to the form represented by the $\mathrm{KN}$ model. However, we shall still loosely refer to the nonlinearity represented by the CLL model as self-steepening in this paper. 
For the NLS model, a rogue wave can be interpreted as a long-wave limit of a breather. A breather is a pulsating mode and indeed two types of such modes are possible, one periodic in space and the other periodic in time [6], and theoretical analysis of such modes will constitute the main focus of this work. Many ingenious analytical techniques have already been employed in similar investigations. For instance, the Darboux transformation has been utilized to derive a hierarchy of rational solutions to the NLS equation [15]. Rogue waves can also be studied from the perspective of the interactions between a continuous wave and a soliton [16]. Furthermore, the Hirota bilinear method, an effective method in obtaining multisoliton expressions [17], has likewise been applied to the formulation of NLS equation rogue waves [18].

Rogue wave modes of the KN model have been studied theoretically via the Darboux transformations [19,20]. Here instead we shall study the rogue wave modes of an extended version of the CLL model. Three highlights of the present work can be succinctly illustrated here.

(i) In terms of physical mechanisms, the conventional rogue wave mode of the NLS model with a positive linear dispersion occurs only in the self-focusing-positive cubic nonlinearity regime, where a plane wave exhibits modulation instability. Here we demonstrate that a rogue wave is possible even in a medium with negative cubic nonlinearity provided a form of self-steepening nonlinearity of sufficient strength is present.

(ii) Furthermore, the existence of these rogue waves will be demonstrated to be precisely related to the onset of modulation instabilities, highlighting the importance of modulation instability over the sign of the cubic nonlinearity, in sharp contrast with the situation in the conventional NLS equation.

(iii) In terms of theoretical formulation, we employ the Hirota bilinear method to calculate the breather (pulsating) solutions. We then compute the rogue wave modes as a long-wave limit of such breathers, a procedure commonly performed for most equations within the NLS family. The advantage here is that multisoliton-multibreather expressions are known for large classes of integrable evolution equations through the bilinear method. Hence an alternative mechanism for the derivation of rogue wave modes will result.

The plan of the paper can now be described. In Sec. II we first rederive the well-known rogue wave (Peregrine breather) of the NLS equation through the long-wave limit of a breather. In Sec. III the rogue wave mode of the extended CLL equation is then obtained by this procedure. In Sec. IV physical properties of such modes and modulation instability are investigated. The predictions from these theories are correlated to recent experimental data on water waves of finite amplitude in Sec. V. A discussion is given and results are summarized in Sec. VI.

\section{ROGUE WAVES OF THE NONLINEAR SCHRÖDINGER EQUATION AS A LONG-WAVE LIMIT OF A BREATHER}

A breather of the NLS equation with positive linear dispersion and positive cubic nonlinearity $(\sigma>0)$,

$$
i A_{t}+A_{x x}+\sigma A^{2} A^{*}=0,
$$

where $\sigma$ measures the effect of nonlinear focusing in the system, can be obtained by the Hirota bilinear transformation [21]. Basically, one first isolates a plane-wave background similar to a calculation for dark solitons, but a purely imaginary frequency is employed in the phase. Using the transformation ( $D$ is the Hirota operator $[13,21]$ and the parameter $\alpha$ measures the amplitude of the plane-wave background)

$$
\begin{aligned}
A=\alpha & \exp \left(i \sigma \alpha^{2} t\right)\left[1+\frac{g_{1}}{f}\right], \\
& \left(i D_{t}+D_{x}^{2}\right) g_{1} \cdot f+D_{x}^{2} f \cdot f=0, \\
& D_{x}^{2} f \cdot f=\sigma \alpha^{2}\left[g_{1} g_{1}^{*}+f\left(g_{1}+g_{1}^{*}\right)\right],
\end{aligned}
$$

together with the expansion scheme

$$
\begin{aligned}
f= & \exp (p x)+\exp (-p x)+s \exp (\mathrm{i} \omega t+i \zeta) \\
& +s \exp (-i \omega t-i \zeta), \\
g_{1}= & \lambda \exp (i \omega t+i \zeta)+\mu \exp (-i \omega t-i \zeta),
\end{aligned}
$$

straightforward calculations will give

$$
\begin{aligned}
s & =\left[\frac{1}{1+\frac{p^{2}}{2 \sigma \alpha^{2}}}\right]^{1 / 2}, \quad \omega=p \sqrt{p^{2}+2 \sigma \alpha^{2}}, \\
\lambda & =\frac{s p}{\sigma \alpha^{2}}\left(p+\sqrt{p^{2}+2 \sigma \alpha^{2}}\right), \\
\mu & =\frac{s p}{\sigma \alpha^{2}}\left(p-\sqrt{p^{2}+2 \sigma \alpha^{2}}\right) .
\end{aligned}
$$

The parameter $\zeta$, originally coming from the flexibility in choosing the starting point in time, will now be allowed to take on arbitrary values. In particular, on choosing $\exp (i \zeta)=-1$ and taking the limit of $p$ approaching zero, one now arrives at

$$
A=\alpha \exp \left(i \sigma \alpha^{2} t\right)\left\{1-\frac{2\left(1+2 i \sigma \alpha^{2} t\right)}{\sigma \alpha^{2}\left(x^{2}+2 \sigma \alpha^{2} t^{2}+\frac{1}{2 \sigma \alpha^{2}}\right)}\right\},
$$

the familiar Peregrine breather rogue wave solution of the NLS equation. A nonsingular profile is only possible for $\sigma>0$ (positive cubic nonlinearity), consistent with the known results of the NLS equation. This breather has often been derived with similar ease via the Darboux transformation in the literature. However, the treatment of the CLL equation by the Darboux scheme may encounter difficulties and these aspects are discussed in the Appendix.

\section{DERIVATIVE NONLINEAR SCHRÖDINGER MODEL}

Following the reasoning of the previous section, one must first obtain the breather of the extended CLL model, namely,

$$
i A_{t}+A_{x x}-\sigma A^{2} A^{*}+i \gamma A A^{*} A_{x}=0,
$$

through a Hirota bilinear transformation. Physically, the term associated with $\sigma$ measures the cubic nonlinearity. In the optical context, this refers to the change in the refractive index induced by changing light intensity [13]. The coefficient $\gamma$ is loosely related to self-steepening. In optical waveguides, 
this refers to the dependence of the group velocity on light intensity [13]. For convenience in the subsequent theoretical presentation, we have adopted a negative sign in front of the cubic nonlinear term. Here $\sigma$ and $\gamma$ are arbitrary and real valued. Importantly, we can allow for $\sigma>0$, namely, incorporating a negative cubic nonlinearity, in sharp contrast with the conventional NLS theory [Eq. (2) for $\gamma=0$ ], where rogue waves are only possible for positive cubic nonlinearity. Additional insights in terms of modulation instability are then generated. The bilinear transform was historically first given for the bright soliton case [22]. However, a generalized format must be used for the case of dark solitons and breathers, which is given as [23] $A=\frac{G}{F}$, where both $F$ and $G$ may be complex $(C=$ const $)$,

$$
\begin{aligned}
\left(i D_{t}+D_{x}^{2}-C\right) G \cdot F & =0, \\
\left(D_{x}^{2}-C\right) F \cdot F^{*} & =\frac{i \gamma\left(D_{x} G \cdot G^{*}\right)}{2}-\sigma G G^{*}, \\
D_{x} F \cdot F^{*} & =\frac{i \gamma G G^{*}}{2} .
\end{aligned}
$$

Continuous waves and bright and dark solitons can be recovered by an appropriate choice of exponential functions $[22,23]$. Here we calculate the breather (a pulsating mode) by the following expansion scheme and the auxiliary functions $f$ and $g$ defined through the parameters $p, M$, and $\rho_{0}$ (all real valued); $a_{n}, b_{n}$, and $\Omega$ (all complex valued); and $\zeta^{(1)}$ and $\zeta^{(2)}$ (complex-valued phase factors):

$$
\begin{aligned}
G= & \rho_{0} \exp (-i \omega t)\left\{1+a_{1} \exp \left(i p x-\Omega t+\zeta^{(1)}\right)\right. \\
& +a_{2} \exp \left(-i p x-\Omega^{*} t+\zeta^{(2)}\right) \\
& \left.+M a_{1} a_{2} \exp \left[-\left(\Omega+\Omega^{*}\right) t+\zeta^{(1)}+\zeta^{(2)}\right]\right\} \\
= & \rho_{0} \exp (-i \omega t) g, \\
F= & \exp (-i \beta x)\left\{1+b_{1} \exp \left(i p x-\Omega t+\zeta^{(1)}\right)\right. \\
& +b_{2} \exp \left(-i p x-\Omega^{*} t+\zeta^{(2)}\right) \\
& \left.+M b_{1} b_{2} \exp \left[-\left(\Omega+\Omega^{*}\right) t+\zeta^{(1)}+\zeta^{(2)}\right]\right\} \\
= & \exp (-i \beta x) f, \\
\beta= & -\frac{\gamma \rho_{0}^{2}}{4}, \quad \omega=\sigma \rho_{0}^{2}-\frac{3 \gamma^{2} \rho_{0}^{4}}{16} .
\end{aligned}
$$

The bilinear equations then dictate that

$$
\begin{aligned}
a_{1} & =-b_{1}\left(\frac{p^{2}-2 \beta p-i \Omega}{p^{2}+2 \beta p+i \Omega}\right), \\
a_{2} & =-b_{2}\left(\frac{p^{2}+2 \beta p-i \Omega^{*}}{p^{2}-2 \beta p+i \Omega^{*}}\right), \\
b_{2}^{*}\left(\frac{p^{2}+2 \beta p-i \Omega}{p^{2}-2 \beta p-i \Omega}\right) & =b_{1}\left(\frac{p^{2}-2 \beta p+i \Omega}{p^{2}+2 \beta p+i \Omega}\right) .
\end{aligned}
$$

For the calculations of the dark soliton, one needs a real wave number (in the spatial variable $x$ ) and a real frequency (in the time variable $t$ ). In the optical fiber setting, the roles of $x$ and $t$ as space and time are reversed. For the present case of a breather, a purely imaginary wave number is needed. More importantly, the angular frequency $\Omega$ must be complex and will satisfy a quadratic dispersion relation

$$
\Omega^{2}+4 \beta p i \Omega+p^{4}-12 \beta^{2} p^{2}+2 \sigma \rho_{0}^{2} p^{2}=0 .
$$

The threshold condition for the existence of such breathers is that this angular frequency cannot be purely imaginary (otherwise a singular solution will occur) and must be genuinely complex, i.e., the condition on the discriminant of the quadratic dispersion should be

$$
8 \beta^{2}-p^{2}-2 \sigma \rho_{0}^{2}>0 .
$$

Using Eq. (4) and the anticipation of a long-wave limit ( $p \rightarrow$ 0 ) in the rogue wave calculation, Eq. (6a) implies that

$$
4 \sigma<\gamma^{2} \rho_{0}^{2} .
$$

When $\sigma \leqslant 0$ (the NLS equation with positive cubic nonlinearity and positive linear dispersion), this constraint is always satisfied. However, for $\sigma>0$ (negative cubic nonlinearity), the self-steepening nonlinearity must attain a certain strength, relative to the negative cubic nonlinearity and the amplitude of the background plane wave, to sustain the rogue wave mode. The surprising coincidence is that Eq. (6b) is the precise condition for the onset of modulation instability.

Returning to the breather calculations, Eqs. (5) and (6a) now yield a real constant $M$ in the interaction terms given by

$$
M=1+\frac{4 p^{4}}{\left(\Omega+\Omega^{*}\right)^{2}} .
$$

Following the logic of the NLS case, a long-wave limit $p$ $\rightarrow 0$ is now taken. The asymptotic expansion of the angular frequency is

$$
\Omega=p\left(\Omega_{0}+\Omega_{1} p+\Omega_{2} p^{2}\right)
$$

where $\Omega_{0}$ satisfies

$$
\Omega_{0}\left(\Omega_{0}+4 \beta i\right)=12 \beta^{2}-2 \sigma \rho_{0}^{2} .
$$

Further calculations show that $\Omega_{1}=0$ or $\Omega_{0}=-2 \beta i$. The latter case is inadmissible as it contradicts the discriminant condition (6a). The next order of correction is

$$
\Omega_{2}=-\frac{1}{2\left(\Omega_{0}+2 \beta i\right)} .
$$

The explicit form of the rogue wave mode can now be computed by taking $b_{1}=1$ and phase factors $\exp \left(\zeta^{(1)}\right)=$ $\exp \left(\zeta^{(2)}\right)=-1$ :

$$
\begin{aligned}
f= & 1-b_{1} \exp (i p x-\Omega t)-b_{2} \exp \left(-i p x-\Omega^{*} t\right) \\
& +M b_{1} b_{2} \exp \left[-\left(\Omega+\Omega^{*}\right) t\right] \\
= & p^{2}\left[x^{2}+\frac{4}{\left(\Omega_{0}+\Omega_{0}^{*}\right)^{2}}+\left|\Omega_{0}\right|^{2} t^{2}+i t x\left(\Omega_{0}-\Omega_{0}^{*}\right)\right. \\
& \left.+\frac{8 \beta\left(\Omega_{0} t-i x\right)}{\left(\Omega_{0}^{*}\right)^{2}+4 \beta^{2}}\right]+O\left(p^{3}\right) \\
= & p^{2} f_{2}+O\left(p^{3}\right) .
\end{aligned}
$$


The coefficient of $p^{2}$ in $f$ can be further simplified algebraically as

$f_{2}=(x-b t)^{2}+a^{2} t^{2}+\frac{1}{a^{2}}-\frac{4 b}{a} \frac{(a+2 b i)[a t+(b t-x) i]}{\left(a^{2}+4 b^{2}\right)}$,

where

$$
\begin{aligned}
\Omega_{0}\left(\Omega_{0}+4 \beta i\right) & =12 \beta^{2}-2 \sigma \rho_{0}^{2}, \quad \Omega_{0}=a+b i, \\
a & = \pm \sqrt{8 \beta^{2}-2 \sigma \rho_{0}^{2}}, \quad b=-2 \beta .
\end{aligned}
$$

The crucial point is to show that this rogue wave mode is nonsingular. The imaginary part of $f_{2}$ is zero only if $x=3 b t$.
However, in that case, the real part is then

$$
a^{2} t^{2}+\frac{(2 a b t-1)^{2}}{a^{2}}
$$

which is positive definite. Hence, $f_{2}$ is nonzero.

Further algebra now leads to the rogue wave mode of Eq. (2) as

$$
A=\rho_{0} \exp (i \beta x-i \omega t) \frac{g_{2}}{f_{2}}
$$

where

$$
\frac{g_{2}}{f_{2}}=1+\frac{(-8 a b i) x+\left[4 a\left(2 b^{2}-a^{2}\right) i\right] t+(-4 a+8 b i)}{a\left(a^{2}+4 b^{2}\right)\left\{(x-b t)^{2}+a^{2} t^{2}+\frac{1}{a^{2}}-\frac{4 b}{a} \frac{(a+2 b i)[a t+(b t-x) i]}{\left(a^{2}+4 b^{2}\right)}\right\}},
$$

with the auxiliary parameters $a, b, \beta$, and $\omega$ given by Eqs. (4) and (11), for $A$ to satisfy Eq. (2). If the cubic nonlinearity $\sigma$ and self-steepening $\gamma$ of the system are given, Eqs. (4) and (11)-(13) constitute a one-parameter $\rho_{0}$ family of exact solutions of Eq. (2).

\section{ANALYTICAL PROPERTIES OF THE ROGUE WAVE}

\section{A. Amplitude of the rogue wave}

It will be instructive to examine the analytical properties of the rogue wave mode derived in the preceding section. For the Peregrine breather of the NLS model, the maximum amplitude is three times that of the background value. Despite the complexity of Eqs. (12) and (13), surprisingly, an analytical formulation for the maxima and minima exists. More precisely, the square of the magnitude of the complex expression in Eq. (13), defined by

$$
H(x, t)=\left|\frac{g_{2}}{f_{2}}\right|^{2},
$$

possesses extrema (turning points where the first derivatives vanish) at

$$
\begin{aligned}
(x, t)= & \left(\frac{6 b^{2}}{a^{3}+4 a b^{2}}, \frac{2 b}{a^{3}+4 a b^{2}}\right),\left(\frac{6 b^{2}+a \sqrt{3\left(a^{2}+b^{2}\right)}}{a^{3}+4 a b^{2}}, \frac{2 a^{2} b+2 b^{3}-a b \sqrt{3\left(a^{2}+b^{2}\right)}}{a^{5}+5 a^{3} b^{2}+4 a b^{4}}\right), \\
& \left(\frac{6 b^{2}-a \sqrt{3\left(a^{2}+b^{2}\right)}}{a^{3}+4 a b^{2}}, \frac{2 a^{2} b+2 b^{3}+a b \sqrt{3\left(a^{2}+b^{2}\right)}}{a^{5}+5 a^{3} b^{2}+4 a b^{4}}\right)
\end{aligned}
$$

and hence the local maximum is located at

$$
(x, t)=\left(\frac{6 b^{2}}{a^{3}+4 a b^{2}}, \frac{2 b}{a^{3}+4 a b^{2}}\right) .
$$

The reasons are that (i) the second derivative is negative

$$
\frac{\partial^{2} H}{\partial x^{2}}=-48 a^{2}-448 b^{2}<0
$$

and (ii) the local quadratic form is of a definite sign

$$
\begin{gathered}
\frac{\partial^{2} H}{\partial x^{2}} \frac{\partial^{2} H}{\partial t^{2}}-\left(\frac{\partial^{2} H}{\partial x \partial t}\right)^{2}= \\
+468 a^{6}+9216 a^{4} b^{2}+36864 a^{2} b^{4} \\
+4915 b^{6}>0
\end{gathered}
$$

at that point. The other two extrema are minima as the function attains a value of zero and the configuration is illustrated by Figs. 1-3. At the maximum point, the function $H(x, t)$ attains a value of 9 , proving that the amplitude of the rogue wave is three times that of the background continuous wave (Figs. 1-3).

Although this maximum amplification ratio is fixed, the shape and spatial extent may vary with the two parameters of the problem, namely, the cubic nonlinearity and selfsteepening coefficients, provided their magnitudes permit the existence of the rogue wave, as outlined in the constraints below. More precisely, changing the magnitude of the cubic nonlinearity does not affect the shape and extent of the rogue wave modes drastically (Figs. 1 and 2). However, changing the magnitude of the self-steepening nonlinearity does pose a significant modification of the spatial size of the rogue wave modes (Figs. 1 and 3). 


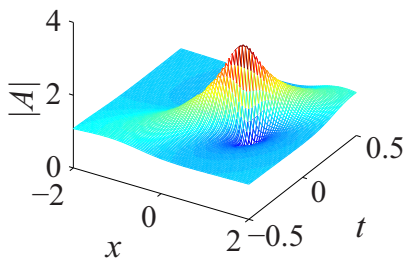

(a)

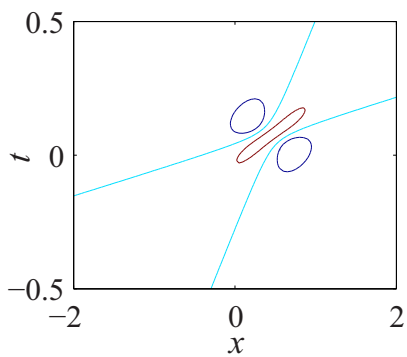

(b)

FIG. 1. (Color online) Plot of the amplitude of the rogue wave mode, $|A|$ of Eqs. (12) and (13), versus $x$ and $t$ for $\sigma=1, \gamma=4$, and $\rho_{0}=1$ : (a) three-dimensional view and (b) two-dimensional contour plot. In (b) the top and bottom ovals represent displacement equal to 0.5 , the middle oval represents displacement equal to 2 , and the two hyperbolic curves represent displacement equal to 1 .

\section{B. Modulation instability and the criterion for the existence of a rogue wave}

Of a much broader significance is the issue of modulation instability. Starting from a plane wave of Eq. (2),

$$
A=\alpha \exp \left\{i \lambda x-i\left[\lambda^{2}+(\sigma+\gamma \lambda) \alpha^{2}\right] t\right\},
$$

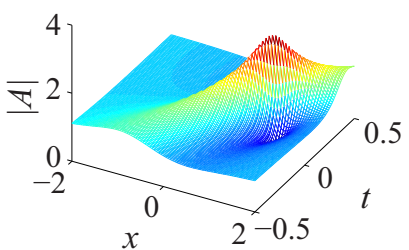

(a)

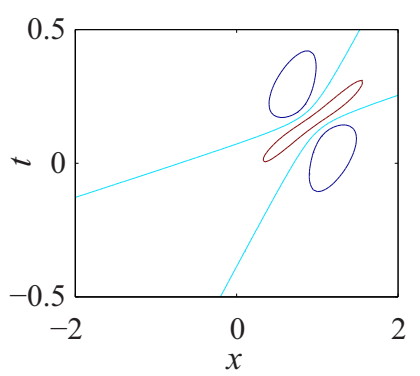

(b)

FIG. 2. (Color online) Plot of the amplitude of the rogue wave mode, $|A|$ of Eqs. (12) and (13), versus $x$ and $t$ for $\sigma=3, \gamma=4$, and $\rho_{0}=1$ : (a) three-dimensional view and (b) two-dimensional contour plot. In (b) the top and bottom ovals represent displacement equal to 0.5 , the middle oval represents displacement equal to 2 , and the two hyperbolic curves represent displacement equal to 1 .

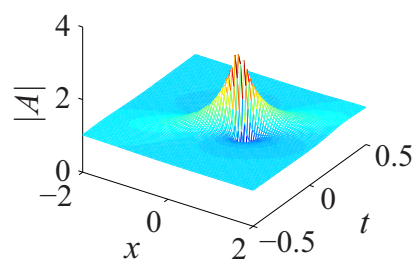

(a)

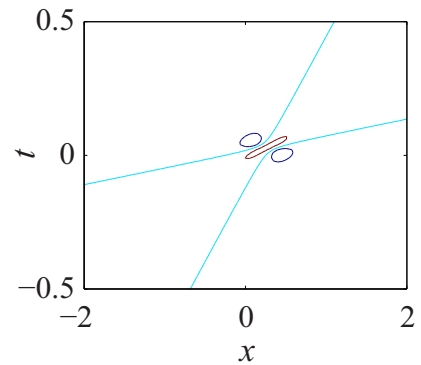

(b)

FIG. 3. (Color online) Plot of the amplitude of the rogue wave mode, $|A|$ of Eqs. (12) and (13), versus $x$ and $t$ for $\sigma=1, \gamma=6$, and $\rho_{0}=1$ : (a) three-dimensional view and (b) two-dimensional contour plot. In (b) the top and bottom ovals represent displacement equal to 0.5 , the middle oval represents displacement equal to 2 , and the two hyperbolic curves represent displacement equal to 1 .

one now imposes small perturbations of the form

$$
\exp [i(r x-s t)] \text {. }
$$

The dispersion relation then yields

$$
\left[s-\left(2 \lambda+\gamma \alpha^{2}\right) r\right]^{2}=r^{2}\left[r^{2}+2(\sigma+\lambda \gamma) \alpha^{2}\right] .
$$

For the rogue wave modes (4) and (11)-(13), one has $\alpha=\rho_{0}$ and $\lambda=-\frac{\gamma \rho_{0}^{2}}{4}$ and thus if

$$
4 \sigma>\gamma^{2} \rho_{0}^{2},
$$

$s$ is always real and there is no modulation instability. However, if

$$
4 \sigma<\gamma^{2} \rho_{0}^{2},
$$

$s$ can be complex and modulation instability can now arise. In a quite remarkable coincidence this is exactly Eq. (6b), the condition for the occurrence of a rogue wave mode.

\section{PHYSICAL APPLICATIONS}

\section{A. Water waves}

The evolution of a weakly nonlinear, slowly varying wave packet in the group velocity frame will be governed by the nonlinear Schrödinger equation to the third order in amplitude. When a fourth-order expansion is invoked, the resulting Dysthe model incorporates a nonlinearity as displayed in Eq. (2), as well as other terms connected with the induced mean flow [8]. The main goal of this section is to show that the CLL derivative NLS equation, as an approximation of the Dysthe equation that ignores the portion involving the mean flow, can still nevertheless reproduce the main features of 


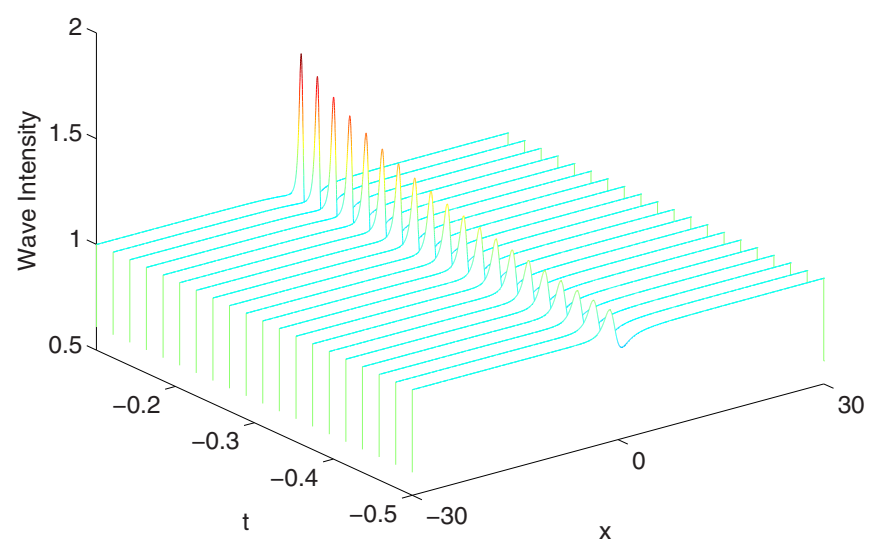

(a)

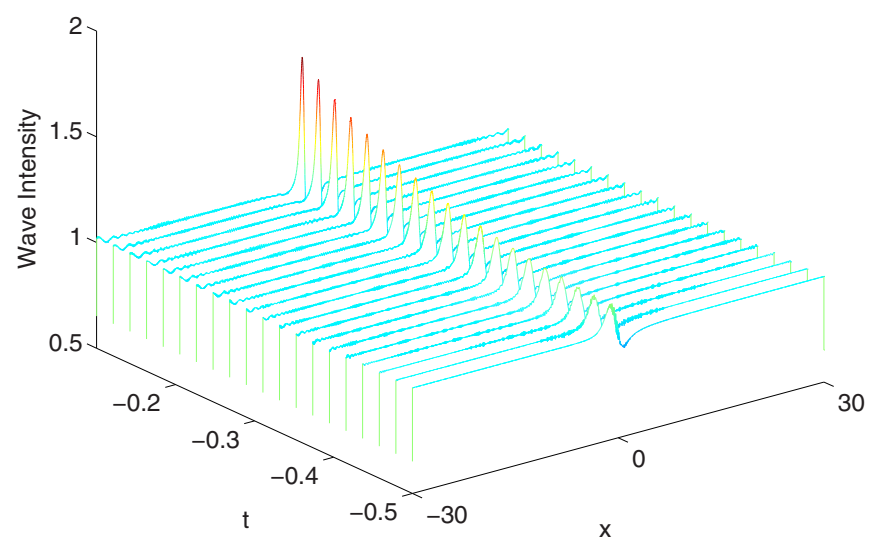

(b)

FIG. 4. (Color online) Growth phase of a rogue wave mode in a parameter regime with finite modulation instability for $\sigma=\rho_{0}=1$, $\gamma=4$, and Eq. (16): (a) plot of the exact solution (12) and (13) and (b) numerical simulation of Eq. (2) with an exact solution perturbed by a $10 \%$ noise as the initial condition.

an experiment conducted recently on finite-amplitude water waves [8]. In that experiment, the evolution of a Peregrine breather of Eq. (1) of Sec. II was measured and compared with numerical simulations of the NLS and Dysthe models. Discrepancies between experimental data and NLS results were found, especially for moderate amplitude, and the Dysthe equation results appear to be better. Here the extended CLL derivative nonlinear Schrödinger equation (2), a significantly simpler model obtained by ignoring the mean flow term, is shown to be consistent with the salient features.

(i) Amplitude of the rogue wave mode. For the Peregrine breather of the NLS model the maximum amplitude of the rogue wave is three times the background. Experimentally, an amplification ratio of slightly less than 3 is recorded even in the finite-amplitude regime. For the extended CLL model, the maximum of Eq. (14) occurs at one of the turning points documented in Eq. (15) and this amplification ratio is still 3, consistent with the observed trend in the laboratory, given the assumption made (i.e., ignoring the mean flow).

(ii) Spectral broadening. Modulation instability produces additional dominant frequencies, which in turn leads to a kind of hydrodynamic supercontinuum. Here the extended

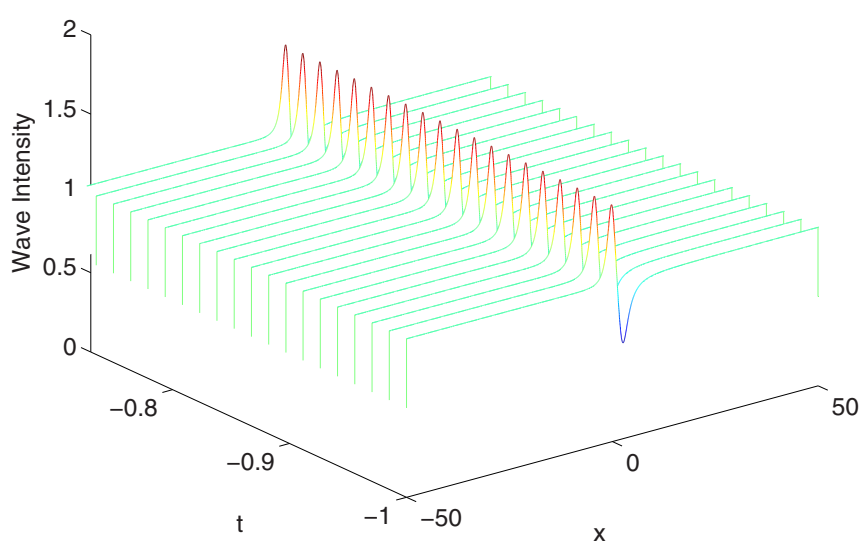

(a)

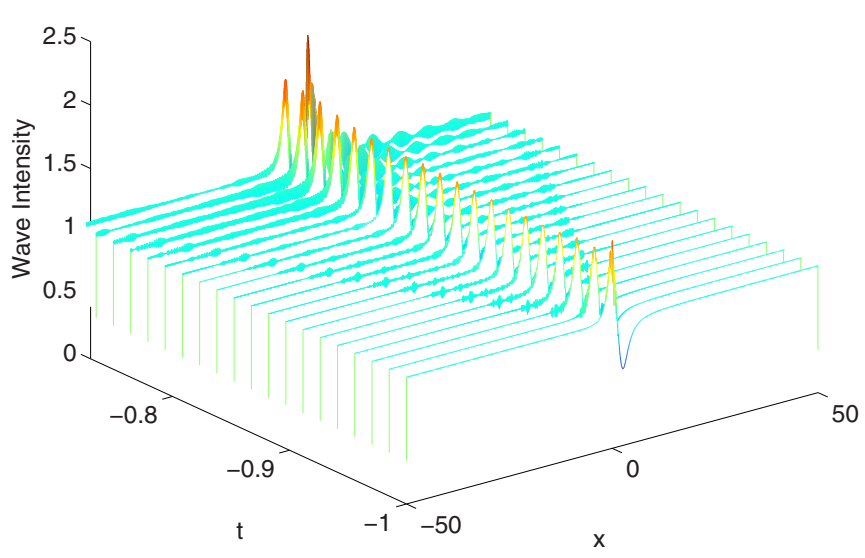

(b)

FIG. 5. (Color online) Growth phase of a rogue wave mode in a parameter regime with marginal modulation instability for $\sigma=\rho_{0}=1, \gamma=2.1$, and Eq. (16): (a) plot of an exact solution (12) and (13) and (b) numerical simulation of Eq. (2) with an exact solution perturbed by a $10 \%$ noise as the initial condition.

CLL derivative NLS equation (2) is shown to possess a wider spectrum of instability modes than the NLS model, explaining why this spectral broadening will occur when a Dysthe-type model (higher-amplitude case) is invoked.

For the purpose of correlation study with the experimental works, we shall adopt the spatial version of the Dysthe equation [Eq. (5) of Ref. [8]], but keep only the self-steepening term. In the present notation, this will read

$$
i A_{t}-A_{x x}-A^{2} A^{*}+8 i \varepsilon|A|^{2} A_{x}=0,
$$

where $\varepsilon$ is an amplitude parameter defined in that experimental study. The plane or continuous wave is

$$
\alpha \exp \left[i \lambda x+i\left(\lambda^{2}-\alpha^{2}-8 \varepsilon \lambda \alpha^{2}\right) t\right] .
$$

Following the standard practice of modulation instability, one now imposes a perturbation, searches for normal modes of the form $\exp [i(r x-s t)]$, and obtains the dispersion relation

$$
\left[s-\left(8 \varepsilon \alpha^{2}-2 \lambda\right) r\right]^{2}=r^{2}\left[r^{2}-2\left(\alpha^{2}+8 \varepsilon \alpha^{2} \lambda\right)\right] .
$$


In the absence of self-steepening (or for the classical NLS model), the range for instability (or complex $s$ ) is

$$
0<r^{2}<2 \alpha^{2},
$$

but, in the presence of self-steepening, this range broadens to

$$
0<r^{2}<2\left(\alpha^{2}+8 \varepsilon \alpha^{2} \lambda\right)
$$

for $\varepsilon>0$ (and $\lambda>0$ ), i.e., a wider band of unstable modes. This trend is exactly what is observed in the experiment.

\section{B. Modulation instability and the growth phase of a rogue wave}

The initial exponential growth of spectral sidebands and the subsequent nonlinear evolution have been discussed theoretically in terms of breathers and in optical experiments through fiber and photonic crystal waveguides [24]. Since the main theme of the present work is to draw a connection between modulation instability (MI) and rogue waves, it will be instructive to examine the occurrence (or to be precise, the growth phase) of a rogue wave at the threshold of MI. For a typical set of parameters far exceeding the threshold of MI $\left(\sigma=\rho_{0}=1\right.$ and $\gamma=4$ [Eq. (6b)]), the growth phase of the rogue wave is robust. Figure 4(a) illustrates the exact solution, while Fig. 4(b) is a numerical marching forward in time using the exact solution plus a $10 \%$ noise as the initial condition. The two figures have a strong resemblance to each other, highlighting the structural stability of a rogue wave in the regime of strong $\mathrm{MI}$. In contrast, in the range of marginal MI $\left(\sigma=\rho_{0}=1\right.$ and $\gamma=2.1$ [Eq. (6b)]), even though an exact solution still exists [Fig. 5(a)], the growth phase of the rogue wave in the presence of a $10 \%$ noise is much less smooth, probably resulting in a smaller likelihood of being observed [Fig. 5(b)].

Finally, it is instructive to consider conceptually the limit of vanishing self-steepening $(\gamma \rightarrow 0)$ in a theoretical attempt to recover the NLS limit. From Eq. (6b), the cubic nonlinearity will also need to become exceedingly small and Eq. (2) degenerates to a purely dispersive wave. Physically, for small self-steepening, the spatial extent of the rogue wave increases dramatically, making it harder to realize in practice.

\section{DISCUSSION AND CONCLUSION}

It has been well established that rogue waves occur only in the regime of positive cubic nonlinearity for a NLS equation with positive linear dispersion, a regime that coincides exactly with the existence of modulation instabilities of plane waves. There are conjectures and numerical evidence that modulation instability is closely related to the onset of rogue wave modes $[24,25]$. The present work provides another strong piece of evidence on this connection. Indeed, we demonstrated that rogue wave modes can occur in a derivative NLS (Chen-LeeLiu) equation with negative cubic nonlinearity, provided a selfsteepening nonlinearity of sufficient strength is also present, and this threshold is shown to correlate exactly with the onset of modulation instability for this derivative NLS equation. It will be useful to examine if such correlation exists for other higher-order NLS equations with third derivatives [16,26], e.g., the Hirota equation.

The precise location and number of rogue wave modes generated will depend on the specific form of the perturbations imposed upon the plane-wave background. Numerical and experimental confirmations can in principle be performed $[27,28]$ and would constitute worthwhile research efforts in the near future. One further challenge in the theoretical analysis is to determine the higher-order rational rogue wave modes for the CLL equation.

Another contribution here is to propose the use of the Hirota bilinear method as an alternative to the Darboux transformation in the computation of a breather mode. This point is particularly valuable if the Darboux scheme encounters technical difficulties and the details are described in the Appendix. Similar to the Darboux case, a long-wave limit in the Hirota formulation is then taken to obtain the rogue wave mode. Many integrable evolution equations possess bilinear forms and extension of this method should allow analytical progress for many other systems. Implementation of this algorithm to other higher-order derivative NLS models is planned for the future. Furthermore, extensions to practical applications, e.g., correlation with marine sea hazards [29], and other branches of physics, e.g., Bose-Einstein condensates [30,31], will be valuable. Indeed, along the line of reasoning of these works, inclusion of the quintic term in the present derivative NLS model, in either a conservative or energy dissipative form, will be a challenging theoretical issue. Such future studies would likely be fruitful.

\section{ACKNOWLEDGMENTS}

D.J.K. acknowledges support from the Australian Research Council (Discovery Project No. DP110102068 and No. DP140100265). Partial financial support for H.N.C. and K.W.C. has been provided by the Research Grants Council General Research Fund Contract No. HKU 711713E.

\section{APPENDIX}

Both the $\mathrm{KN}$ and CLL equations can be represented in Lax pair form as

$$
\begin{aligned}
& R_{x}=U R, \\
& R_{t}=V R
\end{aligned}
$$

where the original derivative nonlinear Schrödinger equations (and conjugate forms) are recovered by the formulations

$$
\begin{aligned}
R_{x t} & =R_{t x}, \\
U_{t}-V_{x}+[U, V] & =0 .
\end{aligned}
$$

In general, such linear representations of a nonlinear system constitute valuable information, indicating integrability and serving as a foundation for several methods to compute exact solutions, e.g., the Darboux scheme. Such procedures have been widely employed in the past on account of their recursive qualities, generating complicated high-order wave functions with relative ease.

The KN equation, expressed as

$$
i A_{t}+A_{x x}+i \gamma\left(|A|^{2} A\right)_{x}=0
$$


is represented by the Lax pair matrices

$$
\begin{aligned}
U & =\left(\begin{array}{cc}
-i \lambda^{2} & \sqrt{\gamma} \lambda A \\
-\sqrt{\gamma} \lambda A^{*} & i \lambda^{2}
\end{array}\right), \\
V & =\left(\begin{array}{cc}
-2 i \lambda^{4}+i \gamma \lambda^{2}|A|^{2} & 2 \sqrt{\gamma} \lambda^{3} A-\sqrt{\gamma^{3}} \lambda|A|^{2} A+i \sqrt{\gamma} \lambda A_{x} \\
-2 \sqrt{\gamma} \lambda^{3} A^{*}+\sqrt{\gamma^{3}} \lambda|A|^{2} A^{*}+i \sqrt{\gamma} \lambda A_{x}^{*} & 2 i \lambda^{4}-i \gamma \lambda^{2}|A|^{2}
\end{array}\right) .
\end{aligned}
$$

The CLL equation, in the form

$$
i A_{t}+A_{x x}+i \gamma|A|^{2} A_{x}=0
$$

is likewise encapsulated by

$$
\begin{aligned}
U & =\left(\begin{array}{cc}
-i \lambda^{2} & \sqrt{\gamma} \lambda A \\
-\sqrt{\gamma} \lambda A^{*} & i \lambda^{2}-\frac{i}{2} \gamma|A|^{2}
\end{array}\right), \\
V & =\left(\begin{array}{cc}
-2 i \lambda^{4}+i \gamma \lambda^{2}|A|^{2} & 2 \sqrt{\gamma} \lambda^{3} A-\frac{1}{2} \sqrt{\gamma^{3}} \lambda|A|^{2} A+i \sqrt{\gamma} \lambda A_{x} \\
-2 \sqrt{\gamma} \lambda^{3} A^{*}+\frac{1}{2} \sqrt{\gamma^{3}} \lambda|A|^{2} A^{*}+i \sqrt{\gamma} \lambda A_{x}^{*} & 2 i \lambda^{4}-i \gamma \lambda^{2}|A|^{2}+\frac{i}{4} \gamma^{2}|A|^{4}+\frac{1}{2} \gamma A_{x} A^{*}-\frac{1}{2} \gamma A_{x}^{*} A
\end{array}\right) .
\end{aligned}
$$

While the two sets of matrices appear to be similar, an elementary Darboux transformation matrix has only been found for the KN case, on account of the clear symmetries existing across and along the matrix diagonals. As a result, breather solutions and their infinite-period limit, the rogue wave, have been uncovered for the $\mathrm{KN}$ equation without significant difficulty.
Unfortunately, the CLL system introduces an asymmetry that complicates matters. A binary Darboux transformation may yet exist, due to the natural pairing of eigenvalues $\lambda$ and $\lambda^{*}$ via the symmetry $U(\lambda)=-U^{\dagger}\left(\lambda^{*}\right)$, but details remain to be worked out. Consequently, we focus on the Hirota bilinear method, which has proven to be an effective alternative here.
[1] K. B. Dysthe, H. E. Krogstad, and P. Müller, Annu. Rev. Fluid Mech. 40, 287 (2008).

[2] A. Chabchoub, N. Hoffmann, M. Onorato, and N. Akhmediev, Phys. Rev. X 2, 011015 (2012).

[3] L. Cavaleri, L. Bertotti, L. Torrisi, E. Bitner-Gregersen, M. Serio, and M. Onorato, J. Geophys. Res. Oceans 117, C00J10 (2012).

[4] D. Clamond, M. Francius, J. Grue, and C. Kharif, Eur. J. Mech. B Fluids 25, 536 (2006).

[5] M. Onorato, A. R. Osborne, M. Serio, L. Cavaleri, C. Brandini, and C. T. Stansberg, Eur. J. Mech. B Fluids 25, 586 (2006).

[6] V. I. Shrira and V. V. Geogjaev, J. Eng. Math. 67, 11 (2010).

[7] A. R. Osborne, Nonlinear Ocean Waves and the Inverse Scattering Transform (Elsevier, Amsterdam, 2010).

[8] L. Shemer and L. Alperovich, Phys. Fluids 25, 051701 (2013).

[9] D. R. Solli, C. Ropers, P. Koonath, and B. Jalali, Nature (London) 450, 1054 (2007).

[10] A. Ankiewicz, J. M. Soto-Crespo, M. A. Chowdhury, and N. Akhmediev, J. Opt. Soc. Am. B 30, 87 (2013).

[11] H. H. Chen, Y.C. Lee, and C. S. Liu, Phys. Scr. 20, 490 (1979).

[12] D. J. Kaup and A. C. Newell, J. Math. Phys. 19, 798 (1978).

[13] Y. S. Kivshar and G. P. Agrawal, Optical Solitons: From Fibers to Photonic Crystals (Academic, New York, 2003).

[14] S. Kakei, N. Sasa, and J. Satsuma, J. Phys. Soc. Jpn. 64, 1519 (1995).

[15] D. J. Kedziora, A. Ankiewicz, and N. Akhmediev, Phys. Rev. E 88, 013207 (2013).

[16] G. Yang, L. Li, and S. Jia, Phys. Rev. E 85, 046608 (2012).
[17] Y. Matsuno, The Bilinear Transformation Method (Academic, New York, 1984).

[18] Y. Ohta and J. Yang, Proc. R. Soc. London Ser. A 468, 1716 (2012).

[19] S. Xu, J. He, and L. Wang, J. Phys. A: Math. Theor. 44, 305203 (2011).

[20] B. Guo, L. Ling, and Q. P. Liu, Stud. Appl. Math. 130, 317 (2013).

[21] K. W. Chow, J. Phys. Soc. Jpn. 64, 1524 (1995).

[22] A. Nakamura and H. H. Chen, J. Phys. Soc. Jpn. 49, 813 (1980).

[23] D. W. C. Lai and K. W. Chow, J. Phys. Soc. Jpn. 70, 666 (2001).

[24] J. M. Dudley, G. Genty, F. Dias, B. Kibler, and N. Akhmediev, Opt. Express 17, 21497 (2009).

[25] D. R. Solli, C. Ropers, and B. Jalali, Nonlinearity 26, R85 (2013).

[26] Y. Tao and J. He, Phys. Rev. E 85, 026601 (2012).

[27] A. Slunyaev, E. Pelinovsky, A. Sergeeva, A. Chabchoub, N. Hoffmann, M. Onorato, and N. Akhmediev, Phys. Rev. E 88, 012909 (2013).

[28] A. Chabchoub, N. Hoffmann, M. Onorato, A. Slunyaev, A. Sergeeva, E. Pelinovsky, and N. Akhmediev, Phys. Rev. E 86, 056601 (2012).

[29] E. Pelinovsky, I. Didenkulova, F. Mendez, D. Rybski, and S. Tinti, Nat. Hazards Earth Syst. Sci. 13, 1063 (2013).

[30] Y. V. Bludov, V. V. Konotop, and N. Akhmediev, Phys. Rev. A 80, 033610 (2009).

[31] Z. Yan, V. V. Konotop, and N. Akhmediev, Phys. Rev. E 82, 036610 (2010). 